Published in final edited form as:

Reprod Toxicol. 2018 June ; 78: 40-49. doi:10.1016/j.reprotox.2018.03.007.

\title{
Peripubertal serum dioxin concentrations and subsequent sperm methylome profiles of young Russian adults
}

\author{
J. Richard Pilsner ${ }^{1,{ }^{*}}$, Alex Shershebnev ${ }^{1}$, Yulia Medvedeva ${ }^{2,3}$, Alexander Suvorov $^{1}$, Haotian \\ $\mathbf{W u}^{1}$, Andrey Goltsov ${ }^{4}$, Evgeny Loukianov ${ }^{5}$, Tatiana Andreeva ${ }^{5}$, Fedor Gusev ${ }^{5}$, Andrey \\ Manakhov $^{5}$, Luidmila Smigulina ${ }^{6}$, Maria Logacheva ${ }^{7,8}$, Victoria Shtratnikova ${ }^{7}$, Irina \\ Kuznetsova $^{5}$, Peter Speranskiy-Podobed ${ }^{5}$, Jane S. Burns ${ }^{9}$, Paige L. Williams ${ }^{10,11}$, Susan \\ Korrick $^{9,12}$, Mary M. Lee ${ }^{13}$, Evgeny Rogaev ${ }^{5,14}$, Russ Hauser ${ }^{9,11}$, and Oleg Sergeyev ${ }^{5,6,7, *}$ \\ ${ }^{1}$ Department of Environmental Health Sciences, School of Public Health and Health Sciences, \\ University of Massachusetts, 686 North Pleasant Street, Amherst, MA 01003, USA \\ ${ }^{2}$ Institute of Bioengineering, Research Center of Biotechnology RAS, 60 let Oktjabrja pr-t, 7, bld. \\ 1, 117312, Moscow, Russia
}

${ }^{3}$ Department of Computational Biology, Vavilov Institute of General Genetics, Russian Academy of Sciences, 3 Gubkina St., 119991, Moscow, Russia

${ }^{4}$ Department of Molecular Genetics, Research Center for Obstetrics, Gynecology and Perinatology, 4 Oparin St., 117997, Moscow, Russia

${ }^{5}$ Department of Genomics and Human Genetics, Vavilov Institute of General Genetics, Russian Academy of Sciences, 3 Gubkina St., 119991, Moscow, Russia

${ }^{6}$ Chapaevsk Medical Association, 3a Meditsinskaya st., 446100, Chapaevsk, Samara region, Russia

${ }^{7}$ A.N. Belozersky Research Institute of Physico-Chemical Biology, Moscow State University, Leninskye gory, house 1, building 40, 119992, Moscow, Russia

${ }^{8}$ Skolkovo Innovation Center, Building 3, 143026, Moscow, Russia

${ }^{9}$ Environmental and Occupational Medicine and Epidemiology Program, Department of Environmental Health, Harvard T.H. Chan School of Public Health, 665 Huntington Avenue, Boston, Massachusetts, 02115, USA

${ }^{10}$ Department of Biostatistics, Harvard T.H. Chan School of Public Health, 665 Huntington Avenue, Boston, Massachusetts, 02115, USA

\footnotetext{
*Corresponding Authors: Oleg Sergeyev, A.N. Belozersky Research Institute of Physico-Chemical Biology, Moscow State University, Leninskye gory, house 1, building 40, 119992, Moscow, Russia. Telephone: +7 84639-22311, Cell: +7 9277465369, Fax: +7 84639-22311, olegsergeyev1@yandex.ru, J. Richard Pilsner, PhD, MPH, Department of Environmental Health Sciences, School of Public Health and Health Sciences, University of Massachusetts Amherst, 1471A Goessmann, 686 North Pleasant Street, Amherst, MA 01003, USA, Tel: 413-545-0036, rpilsner@umass.edu.

Publisher's Disclaimer: This is a PDF file of an unedited manuscript that has been accepted for publication. As a service to our customers we are providing this early version of the manuscript. The manuscript will undergo copyediting, typesetting, and review of the resulting proof before it is published in its final citable form. Please note that during the production process errors may be discovered which could affect the content, and all legal disclaimers that apply to the journal pertain.

Disclosures: None of the authors has any actual or pending competing financial interests.
} 
${ }^{11}$ Department of Epidemiology, Harvard T.H. Chan School of Public Health, 665 Huntington Avenue, Boston, Massachusetts, 02115, USA

${ }^{12}$ Channing Division of Network Medicine, Brigham and Women's Hospital, Harvard Medical School, 181 Longwood Avenue, Boston, Massachusetts, 02115, USA

${ }^{13}$ Pediatric Endocrine Division, Department of Pediatrics, University of Massachusetts Medical School, 55 N Lake Avenue, Worcester, Massachusetts, 01655, USA

${ }^{14}$ Department of Psychiatry, Brudnick Neuropsychiatric Research Institute, University of Massachusetts Medical School, 303 Belmont Street, 01604 Worcester, Massachusetts, USA

\section{Abstract}

Background-The association of exposure to endocrine disrupting chemicals in the peripubertal period with subsequent sperm DNA methylation is unknown.

Objective-We examined the association of peripubertal serum 2,3,7,8-tetrachlorodibenzo-pdioxin (TCDD) concentrations with whole-genome bisulfite sequencing (WGBS) of sperm collected in young adulthood.

Methods-The Russian Children's Study is a prospective cohort of 516 boys who were enrolled at 8-9 years of age and provided semen samples at 18-19 years of age. WGBS of sperm was conducted to identify differentially methylated regions (DMR) between highest $(n=4)$ and lowest $(\mathrm{n}=4)$ peripubertal TCDD groups.

Results-We found 52 DMRs that distinguished lowest and highest peripubertal serum TCDD concentrations. One of the top scoring networks, "Cellular Assembly and Organization, Cellular Function and Maintenance, Carbohydrate Metabolism", identified estrogen receptor alpha as its central regulator.

Conclusion-Findings from our limited sample size suggest that peripubertal environmental exposures are associated with sperm DNA methylation in young adults.

\section{Keywords}

peripuberty; susceptible window of germ cell development; endocrine disrupting chemicals; environmental effects; whole genome bisulfite sequencing; DNA methylation; sperm methylome; Russian Children's Study

\section{Introduction}

Compelling animal data in the past few years have demonstrated that the epigenome of the male germ cell is sensitive to environmental conditions at specific windows of development that can affect the phenotype of future generations [1]. Most notably, in utero exposures to endocrine disrupting chemicals (EDCs), including exposures to dioxins [2], have been shown to elicit inter- [3] and transgenerational [4-7] changes in sperm DNA methylation. More recently, it has been recognized that during spermatogenesis, the epigenome in adult animals is also susceptible to environmental conditions such as low protein diet [8] and prediabetic conditions [9]. 
Another susceptible window of germ cell development is during peripuberty; however, no data exist on the effects of environmental exposures on the sperm epigenome during this period. The male peripubertal period in humans comprises of 1-2 years prior to the first signs of pubertal onset (testicular volume $>3 \mathrm{ml}$, Tanner genitalia and pubarche staging G2 and P2) followed by activation of the hypothalamus-pituitary-gonadal (HPG) axis [10,11] and the proliferation of Sertoli cells and spermatogonia [12]. It has been proposed that the awakening of the HPG axis during the peripubertal period primes the male reproductive system prior to full activation at puberty [1]. Given that this process is hormone-dependent, exposure to EDCs during this period could reprogram the trajectory of male reproductive capacity throughout the lifecourse [1].

Dioxins are a group of structurally-related organochlorines that are generated as unwanted by-products of chemical industrial manufacturing processes and incineration. Due to their resistance to degradation and high lipophilicity, dioxins have caused widespread environmental contamination and bioaccumulate in the food chain [13-15], resulting in continued human exposure through consumption of meat, dairy products, and fish [16]. 2,3,7,8-tetrachlorodibenzo-p-dioxin (TCDD) is the most toxic dioxin congener with known endocrine disrupting properties. Our previous data showed that peripubertal serum TCDD concentrations were associated with subsequent poor semen parameters, including sperm concentration, total sperm count and motile sperm, in early adulthood [17]. Such results were also found with childhood (age 1-9 years) serum TCDD concentrations [18]. Known effects of dioxins on male reproductive health and epigenetic changes induced by dioxins are reviewed elsewhere [19].

Given the compelling data suggesting that environmental conditions during windows of germ cell development can alter sperm epigenetics, we conducted a prospective cohort study of Russian young men with serum dioxin-like compounds concentrations measured at ages 8-9 years to examine the association of peripubertal serum TCDD concentrations with subsequent profiles of sperm DNA methylation via whole genome bisulfite sequencing (WBGS) from semen samples collected in early adulthood.

\section{Methods}

\subsection{Study population}

The Russian Children's Study (RCS) is a prospective cohort of 516 boys in Chapaevsk who were enrolled from 2003-2005 at 8 to 9 years of age [20,21]. After study enrollment, they were followed annually through 19 to 22 years of age. The enrollment rate was high, with 90\% of all eligible boys in Chapaevsk participating. During each annual follow-up visit, physical examinations including anthropometry, pubertal staging and testicular volume measurements were performed. Blood samples for chemical, hormonal, genetic and epigenetic analysis were collected at baseline and biennially. Cumulatively, more than 4700 visits have been conducted as of February 2017. Subjects who reached sexual maturity and were at least 18 years of age were invited to provide two semen samples approximately one week apart. 
For the current study, we selected subjects from the cohort of 516 young men who had the following existing data/samples: peripubertal serum TCDD measurements at 8-9 years old, frozen semen and leukocyte samples collected at 18-19 years old and semen quality parameters. To investigate the epigenetic changes in sperm samples with maximal differences in peripubertal TCDD concentrations, of 147 eligible subjects, we selected 4 subjects among those with the highest $\left(95^{\text {th }}-99^{\text {th }}\right.$ percentiles) and 4 among those with the lowest (level below limit of detection) peripubertal serum TCDD concentrations. We found no significant differences for most of life-style factors including alcohol consumption and nutrition as well as semen parameters by subject selection status in the current study (Supplementary Material, Table S1). Individuals from the selected group did not smoke in the prior year before semen collection in comparison with 61 (64\%) individuals who smoked from non-selected group (P-value $\leq 0.05$ ). Moreover, no significant differences were found in lifestyle factors between the lowest and highest serum TCDD groups. The study was approved by the Human Studies Institutional Review Boards of the Chapaevsk Medical Association (Chapaevsk, Russia); Harvard T. H. Chan School of Public Health, the University of Massachusetts Medical School (Worcester, MA), and Vavilov Institute of General Genetics (Moscow, Russia). At enrollment, the parent or guardian signed an informed consent, and each boy signed an assent before participation. At age 18 or older, the young man signed a consent form prior to providing the semen samples.

\subsection{Dioxin measurements}

During the initial visit when boys were 8-9 years old, fasting blood samples were collected. The serum fraction was stored at $-35^{\circ} \mathrm{C}$ until shipment for analysis at the National Center for Environmental Health at the Centers for Disease Control and Prevention (CDC, Atlanta, GA, USA) and 498 serum samples were analyzed during 2004-2008. Analytes measured included 7 polychlorinated dibenzo-p-dioxins (PCDDs, or dioxins) as described previously [22]. Congener concentrations below the limit of detection (LOD) were assigned the samplespecific LOD divided by the square root of 2 [23]. For 18.9\% subjects with TCDD levels below the LOD, their assigned concentrations ranged from 0.28 to $0.57 \mathrm{pg} / \mathrm{g}$ lipid.

\subsection{Semen collection and semen parameter analyses}

The procedures for semen collection and parameter analyses have been described in detail previously [17]. Briefly, two semen samples were provided by masturbation in a study room near the andrology laboratory one week apart and stored at $37^{\circ} \mathrm{C}$ until semen evaluation, which occurred within 1 hour after ejaculation. Abstinence period was calculated from the dates and times between previous ejaculation and the semen sample obtained for the study. Semen parameter analyses were performed at the Chapaevsk andrology laboratory according to the criteria recently updated [24] by the Nordic Association for Andrology (NAFA) and European Society of Human Reproduction and Embryology - Special Interest Group in Andrology (ESHRE-SIGA) [25]. All samples were assessed by one technician (LS) who was blinded to the serum TCDD concentration. At least 200 sperm per slide were classified within four World Health Organization (WHO) classes: rapidly progressive motile (class a), slowly progressive motile (class b), locally motile (class c) or immotile (class d), using the average percent motile value for duplicate measures [26]. Percent motile sperm was defined as the sum of WHO classes a, b and c. 


\subsection{Isolation of sperm and DNA extraction}

The second semen sample was used for DNA methylation analysis. Upon completion of the semen evaluation, the remaining semen was aliquoted into cryovials and stored at $-80 \mathrm{C}$ without cryoprotectant. Thawed semen samples were subsequently processed at laboratory of evolutionary genomics, Vavilov Institute of General Genetics, Russia with a $20 \mathrm{~min}$ liquefaction at $37 \mathrm{C}$, centrifuged to separate the seminal plasma and the pellet washed in the Sperm Washing Medium (SWM, Irvine Scientific, USA) to decrease viscosity and remove the damaged sperm and somatic cells. A $90 \%$ and $50 \%$ density gradient centrifugation step (90\% and 50\% Isolate, Irvine Scientific, USA) in an Eppendorf 5810® centrifuge with bucket rotor A-4-44 and adapter for 1.5-2.0 ml removed somatic cell contamination and fractionated sperm by their density to upper and bottom layers. Sperm from both layers were merged and sperm DNA was extracted using our newly developed method that homogenizes sperm in the presence of $0.2 \mathrm{~mm}$ stainless steel beads and commercially available reagents [27].

\subsection{Whole genome bisulfite sequencing (WGBS), reduced representation bisulfite sequencing (RRBS), and data processing}

WGBS libraries preparation and sequencing were performed at the Laboratory of Evolutionary Genomics, Vavilov Institute of General Genetics RAS. Libraries for WGBS were constructed using 100-150 ng of DNA and TruSeq DNA methylation kit (Illumina) as per the manufacture's guidelines. Quality control of WGBS libraries were assessed by Bioanalyzer (Agilent). Successful libraries were sequenced on HiSeq 2500 (Illumina) with an average of 216 million reads per sample. Reads were mapped to GRCh38 reference genome using Bismark (version 0.16.1) [28] and Bowtie-2 (version 2.2.9) [29]. To comply with ENCODE recommendations [30], we restricted all subsequent analyses to CpGs with $\geq$ $10 \times$ coverage.

For validation, RRBS libraries from five random samples were prepared using $100 \mathrm{ng}$ of MSPI digested DNA and the Ovation RRBS Methyl-Seq System (Cat \# 0353, Nugen, San Carlos, CA) following standard protocol. Similar to the WGBS, quality and purity of the libraries were assessed via Bioanalyzer and the concentration of the libraries was measured by Qubit fluorometer and verified by real time PCR. cDNA libraries were single-end sequenced using Illumina Hi-Seq 2000 (Illumina, San-Diego, CA) at the Laboratory of Evolutionary Genomics, A.N. Belozersky Research Institute of Physico-Chemical Biology, Moscow State University.

\subsection{Detection of differentially methylated regions (DMRs)}

To identify DMRs which distinguished between the lowest and highest peripubertal TCDD concentrations, we employed MethPipe [31], which uses beta-binomial regression for DMR calling between two groups of samples. The analysis pipeline was employed as described in the software manual. Briefly, bam files produced by Bismark [28] were analyzed to obtain methylation levels at each cytosine site for both strands. Due to the symmetrical nature of $\mathrm{CpG}$ dinucleotides, each $\mathrm{CpG}$ pair produced by methcounts was merged using symmetriccpgs, which also filtered out all mutated sites, detected by methcounts, and non-CpG-context cytosines. Methylation counts for $\mathrm{CpGs}$ for each sample were then merged into a proportion 
table using merge-methcounts with -t flag. The table obtained was used for beta-binomial regression analysis with the help of radmeth program (regression option). Afterwards, each $\mathrm{CpG}$ site was adjusted based on the neighboring sites and the final file with every $\mathrm{CpG}$ containing adjusted p-value and FDR-corrected q-value was produced using radmeth with adjust option with the default values for bins (1:200:1). For the final step in DMR calling, radmeth with merge option was used to merge individually differentially methylated CpGs $(\mathrm{q}<0.05)$ into differentially methylated regions (DMRs).

\subsection{DMR annotation}

All DMR spatial annotations with regards to various genome structures were produced using the closest-features (v. 2.4.25) tool from bedOps package [32]. Gene coordinates were obtained from GENCODE v24 using UCSC Table Browser [33]. For promotors, 2kbp regions around the gene transcription start site (1500bp upstream to 500bp downstream) were used. Enhancer location data were downloaded from FANTOM5 [34,35]. Files containing genomic intervals corresponding to transcription factor binding sites were collected from the ENCODE download section of the UCSC genome browser [36,37]. Nucleosome positional information obtained from MNase-seq experiments in sperm was available as part of GEO submission GSE74426 [38]. For some of the data, liftover from hg19 to hg38 was required and was done online using the UCSC web-based liftOver tool.

\subsection{DMR clustering}

Clustering was performed using clustermap function from seaborn package (v0.7.0) [https:// zenodo.org/record/54844\#.WMlzuxLyuL8] with euclidian distance as a metric.

\subsection{Functional enrichment analyses}

The list of annotated DMRs (Supplementary Material; Table S5) was uploaded to Ingenuity Pathway Analysis (IPA; Qiagen, Hilden, Germany) for core analysis with default settings.

\subsection{Statistical analyses}

Differences in age, anthropometric and semen parameters were evaluated using a two-sided Wilcoxon rank-sum test, Stata software package version 13.1 (StataCorp LP, USA). Differences in the average and distribution of methylation across all $\mathrm{CpG}$ sites between the highest and lowest TCDD concentrations were assessed by t-test and Kolmogorov-Smirnov (KS) tests, respectively. For enrichment analyses, $2 \times 2$ contingency tables were created and Fishers Exact test was used to determine significant enrichment of genomic loci of interest between TCDD groups. KS and Fishers Exact tests were performed using scipy package (v0.18.0) [39] and statistical significance was determined using a threshold of $\mathrm{p}<0.05$.

\section{Results}

We performed whole genome bisulfite sequencing (WGBS) on eight individuals to examine the association of peripubertal ( $8-9$ years of age) serum TCDD concentrations with subsequent sperm methylation profiles as young adults (ages 18-19 years). The mean peripubertal serum TCDD concentrations for the highest and lowest groups were $10.4 \mathrm{pg} / \mathrm{g}$ lipid (range: $8.0-12.1$ ) and $0.39 \mathrm{pg} / \mathrm{g}$ lipid (range: 0.35-0.49), respectively. Table 1 also 
provides mean age, anthropometrics, testicular volume and semen parameters stratified by exposure group. Between the two groups the only significant difference was between serum TCDD concentrations and total TEQ. Individual-level, demographic and clinical data for the eight participants can be found in Supplementary Material; Table S2.

The mean bisulfite conversion efficiency, total unique reads and percent alignment did not differ between groups (see Supplementary Material; Table S3). Restricting data analyses to CpG sites with $\geq 10 \times$ coverage depth, the mean methylation across all $\mathrm{CpG}$ sites were significantly lower in the highest serum TCDD group compared to the lowest group (49.1 $\pm 4.9 \%$ and $61.9 \pm 5.2 \%$, respectively; t-test, $\mathrm{p}=0.01$ ). Additionally, site-specific analyses identified 666 individual CpGs differently methylated between highest versus lowest TCDD groups ( $\geq 10 \%$ methylation change and $\mathrm{q}<0.05$; see Supplementary Material; Table S4).

Since methylation status across regions likely has more pronounced effects on gene expression than methylation at a single $\mathrm{CpG}$, we focused on identifying DMRs that distinguished between lowest and highest peripubertal serum TCDD groups. Restricting to regions with the following parameters ( $\geq 10 \times$ coverage depth, $\geq 3$ CpGs per cluster, $\geq 10 \%$ methylation change and q-value $<0.05$ ), we identified 52 DMRs. Figure 1a shows an unsupervised hierarchical clustering and heatmap of the mean DMR methylation for each individual by TCDD group (also see Supplementary Material; Table S5). Consistent with our findings of mean methylation across all CpG sites, the highest serum TCDD group displayed a lower overall distribution of methylation across the 52 DMRs (Figure 1b, lowest TCDD: median (interquartile range (IQR)): 63.2\% (26.2\%); highest TCDD: 49.1\% (36.3\%); KS test, $\mathrm{p}=0.006$ ), such that $75 \%$ of the DMRs were hypomethylated in highest compared to lowest serum TCDD group (Figure 1c). Interestingly, while methylation across the genome follows a bimodal distribution (e.g., $<20 \%$ or $>80$ methylation), the majority (71.5\%) of DMRs associated with peripubertal serum TCDD were found to have intermediate levels of methylation (20-80\%) (Figure 1d).

To better understand the functional genomics of our peripubertal serum TCDD-associated sperm DMRs, we next investigated DMRs based on their genomic locations. The majority of sperm DMRs were located in gene bodies (55.8\%) and intergenic regions (38.5\%), while $16.2 \%$ and $1.2 \%$ were located in promoters and enhancers, respectively (Figure 2a). Previous research has indicated that nucleosome-associated regions of sperm DNA, as compared to protamine-associated regions, may be important for embryonic development $[40,41]$. Therefore, we utilized publicly available mnase-seq data [38] to determine the overlap of detected sperm DMRs with nucleosome positioning. Overall, only 5 of 52 DMRs were associated with nucleosomes (see Supplementary Material; Table S6). Because the DMR calling software, MethPipe, used for our analyses builds methylation regions for significant DMRs only, we further focused our nucleosome analyses on individual $\mathrm{CpG}$ sites that were differently methylated between lowest and highest serum TCDD groups ( $\mathrm{N}=666$ CpG sites; q-value < 0.05; see Supplementary Material; Table S4). Interestingly, when examining $\mathrm{CpG}$ sites associated with nucleosome regions, we found that the distribution of methylation differed between groups, such that methylation in the highest serum TCDD group displayed a bimodal distribution (e.g., gain and loss of methylation) compared to the lowest group (median (IQR); $58.8 \%$ (58.9\%) vs. 61.7\% (20.5\%), respectively; KS test, $\mathrm{p}=$ 
0.002; Figure 2b). However, in protamine-associated CpGs, the highest TCDD group had a pronounced loss of methylation compared to the lowest group (median (IQR): $47.8 \%$ $(32.9 \%)$ vs. $70.6 \%(31.2 \%)$, respectively; KS test, $\mathrm{p}=1.9 \times 10^{-26}$; Figure $2 \mathrm{c}$ ). Moreover, nucleosome-bound regions of the sperm genome were also highly enriched in significant CpGs (nucleosomes $=0.342 \%$; protamines $=0.085 \%$; Fisher's exact test, $\mathrm{p}=4.4 \times 10^{-25}$ ). Figure 3 provides visualization of individual- and group-level percent methylation for two genes containing DMRs; the nucleosome-associated DMR located in SAMD11 (CpGs = 20), Figure 3a, and the protamine-associated DMR located in PIAS4 (CpGs $=8)$, Figure $3 \mathrm{~b}$.

Functional enrichment analysis of genes proximal to the 52 sperm DMRs associated with peripubertal serum TCDD identified multiple biological networks (Table 2 and Supplementary Materials; Table S7). The top scoring networks included those associated with "Cell Morphology, Hematopoiesis, Humoral Immune Response", "Cellular Assembly and Organization, Cellular Function and Maintenance, Carbohydrate Metabolism" and "DNA Replication, Recombination, and Repair, Energy Production, Nucleic Acid Metabolism". Other enriched networks included those related to development such as "Embryonic Development, Organismal Development, Tissue Development" and "PostTranslational Modification, Cellular Movement, Respiratory System Development and Function". Interestingly, one of our top scoring networks showed the estrogen receptor alpha (ERa/ESR1) as the central regulator under the function of "Cellular Assembly and Organization, Cellular Function and Maintenance, Carbohydrate Metabolism” (Figure 4). Graphical representations of other top networks are found in supplementary material; Figure S1.

Lastly, to validate our WGBS findings, we conducted RRBS on five randomly selected samples and compared 14 DMRs that had overlapping data from both WGBS and RRBS. The majority of the clusters showed a high concordance of DNA methylation between the two platforms (Supplementary Material; Figure S2 and Table 8), demonstrating the validity and reliability of the WGBS results.

\section{Discussion}

To our knowledge, our study is the first to investigate the influence of peripubertal environmental exposures on subsequent sperm DNA methylation in humans. In doing so, we leveraged a long-standing prospective cohort based on $90 \%$ of eligible boys from one city, the Russian Children's Study (RCS), which recruited boys during the peripuberital period (8-9 years old) who had a wide range of serum TCDD concentrations [22]. WGBS of sperm isolated from semen samples collected as young adults (18-19 years old) identified 52 significant DMRs that distinguished four subjects selected from those with the lowest and four subjects selected from those with the highest peripubertal serum TCDD concentrations in this population-based male cohort. The majority of the TCDD-associated sperm DMRs were located in genes or intergenic regions, were hypomethylated, and were highly enriched in previously identified nucleosome-retained regions of the sperm genome. Finally, the top networks generated in enrichment analyses of sperm DMRs revealed functions primarily related to development, cellular organization and DNA replication. 
There is increasing experimental evidence that environmental factors encountered by the male during specific windows of germ cell development may alter epigenetic programming, and in turn, affect the phenotype of subsequent progeny [1]. To date, research has focused on the effect of environmental factors on sperm epigenetic programming at two specific windows of male development: in utero programming of primordial germ cells by nutritional manipulation [42,43] and environmental toxicants [3-5]; and adult programming during spermatogenesis by nutritional manipulation $[9,44]$. Although sperm epigenetic research has provided compelling data supporting an epigenetic legacy of environmental factors during these specific windows of development, research has been minimally applied to uncover environmental effects on sperm epigenetics during other sensitive windows of development, such as the peripubertal period.

Postnatal testicular development varies considerably among mammalian species [45]. While testicular development and secondary sexual maturation in rodents begins shortly after birth [46], in humans, after mini-puberty occurred in early postnatal period, the HPG axis has been thought to be relatively quiescent. However, during this latency period, a number of changes occur in the peripubertal testis and reproductive axis. Nocturnal pulses of gonadotropins lead to changes in Sertoli and Leydig cells that lead to early morning secretion of testosterone, declining concentrations of anti-mullerian hormone [47] and germ cell proliferation [48] that has been observed to occur in waves (e.g., 3-8 years and 10 years) [12]. It has been speculated that childhood and/or peripubertal activation of the HPG axis may "prime" spermatogonia proliferation prior to full activation at puberty, thus any disruption (e.g., nutritional or chemical) of the HPG axis during priming may have longterm impacts on sperm development [1].

TCDD, a known EDC, is the most potent of the dioxin congeners vis-à-vis aryl hydrocarbon receptor (AhR) affinity and is part of a family of chemicals that are highly persistent in the environment and in the food chain $[13,14]$. Ongoing prospective cohorts have provided compelling data showing that early-life exposures to TCDD are associated with abnormal male reproductive health endpoints. In Seveso, Italy, an industrial accident in 1976 resulted in the release of high amounts of dioxin in the atmosphere resulting in widespread contamination. A subsequent study found that those exposed at infancy/prepuberty (1-9 years) had decreased sperm count and motility, while those exposed during mid- to latepuberty (10-17 years) had an increase in sperm count and motility. Interestingly, in those individuals with adult exposure (18-26 years), no associations were found [18]. A later report also found that those infants who were exposed through breastfeeding around the time of the accident had decreased sperm count and motility as adults compared to those who were formula-fed or unexposed [49]. While the TCDD concentrations in Seveso were much higher (210 pg TEQ/g lipid versus 2-8 pg TEQ/g lipid) than those in the RCS after chronic exposure, our results mirrored the Seveso findings [17,18,22]. In the RCS, higher peripubertal serum TCDD was associated with delayed pubertal onset [20], sexual maturition [50], and decreased sperm count and motility [17].

Functional enrichment results of DMRs identified ESR1 as the central regulator of one of the top networks, "Cellular Assembly and Organization, Cellular Function and Maintenance, Carbohydrate Metabolism". Of particular relevance, we have previously reported a 
polymorphism in ESR1, which modified the association between peripubertal dioxin concentrations with pubertal onset based on genitalia staging [51]. Although the primary mode of action of TCDD is via the aryl hydrocarbon receptor $(A h R)$, the anti-estrogenic effects of TCDD on ESR1 signaling are well documented [52,53]. Crosstalk between AhR and ESR1 signaling has been reported, whereby TCDD-activated aryl hydrocarbon receptor nuclear translocator (ARNT) complex has been shown to associate with ESR1 and to recruit p300, a histone acetyltransferase important for cellular proliferation and differentiation [54,55]. Estrogens, along with gonadotropins and androgens, provide critical signaling for spermatogenesis. Unlike the ubiquitous expression of ESR2 throughout the male reproductive system, ESR1 is selectively expressed in Leydig cells and the epithelium of efferent ducts, the region bridging the testes and epididymis [56]. ESR 1 knockout mice are infertile and present with low sperm production and impaired sperm maturation and motility [57-59]. Most recently, membrane ESR1, rather than its nuclear counterpart, was found to be essential for normal spermatogenesis, such that loss of membrane ESR1 resulted in reduced sperm production, viability and motility and increased morphological abnormalities [60]. It has been reported that ESR1 is expressed in rat spermatocytes and spermatids [61,62] and a p300-dependent metabolic remodeling occurs in mouse spermatids [63], during which final DNA methylation patterns are established. Although speculative, the observed sperm DMRs associated with higher peripubertal serum TCDD in our study may be related to indirect effects of ESR1 signaling on Leydig cells. Additionally, altered ESR1 signaling may impact the acquisition of final DNA methylation patterns during meiotic divisions of spermatocytes or during clonal expansion of spermatogonia prior to and/or at puberty - the latter could provide an epigenetic legacy of peripubertal TCDD exposure in adulthood [19].

The histone-protamine exchange is the final epigenetic reprogramming step before the developing male germ cells exit the testes [1]. During this period, it is estimated that $90 \%$ of histones are replaced by protamines, which allows for tighter nuclear compaction to facilitate motility of the haploid genome. Histone retention is not randomly distributed throughout the genome but is enriched in regulatory regions of developmental and imprinted genes that may play an important role in embryo development [40,41,64,65], although some conflicting results have been reported in which histone retention were enriched in gene-poor regions of the genome $[66,67]$. Interestingly, the locations of $\mathrm{CpGs}$ that were differentially methylated between lowest and highest serum TCDD groups were found to be highly enriched in genomic regions with reported nucleosome (i.e., histones) occupancy [38]. In comparison to the lowest TCDD group, significant $\mathrm{CpG}$ sites of the highest TCDD group displayed either a gain or loss of methylation. This was in sharp contrast to results of significant $\mathrm{CpGs}$ enriched in protamine regions, whereby a global trend of methylation loss in the highest TCDD group was observed. Previous studies have shown that protaminebound regions are highly methylated in sperm [38,41]. It is uncertain if the hypomethylation of CpGs observed in the highest TCDD group had downstream effects on chromatin remodeling during the histone-protamine exchange; however, future studies warrant such investigation.

The reproductive implications of the TCDD-associated sperm DMRs are unknown. Among the 52 DMRs, methylation differences between the lowest and highest peripubertal TCDD 
groups ranged from $10 \%$ to $54 \%$. Since only one spermatozoid is sufficient for successful fertilization, methylation for a given $\mathrm{CpG}$ site is considered binary or digital [68] and thus, unlike somatic cells, small increases in percent methylation represent a distribution shift in the proportion or the probability of inheritance of that methylated site. For example, a 54\% reduction in methylation in the highest compared to lowest TCDD group equates to a 50\% increase in the probability that fertilization will occur by a spermatozoid that is hypomethylated at that loci. Thus, even modest $10 \%$ changes in sperm methylation may have significant ramifications for subsequent offspring health and development.

The current study has several limitations. First, our small sample size only includes eight individuals who were selected from among those with the highest and lowest serum TCDD concentrations in a population-based male cohort from one Russian community. In this context, we cannot rule out the potential for other factors to account for methylation differences between our two exposure groups. However, both TCDD groups had similar lifestyles such as smoking status, alcohol consumption and nutritional factors. Second, at relatively low depth of coverage (average $\sim 3 x$ ) across the genome and restriction of analyses to those regions with $\geq 10 \times$ depth of coverage, we achieved a mean of $13 \%$ genome-wide coverage, thereby minimizing the loci available for analyses. Additionally, while our study focused on serum TCDD concentrations during the peripubertal period, its long half-life (approximately 7 years) may correlate with TCDD concentrations at other epigenetic susceptible windows of male germ cell development (e.g., differentiation of primordial germ cells and past and current spermatogenesis), which were not taken into account in this study. Previous studies have also found aberrant sperm DNA methylation among individuals with poor sperm quality [69-71] and recent results from our RCS cohort showed an association between peripubertal TCDD serum concentration and poorer semen parameters [17]. Thus, we are unable to rule out the possibility that sperm DNA methylation is not the prime target of TCDD, but rather is a downstream consequence of the influence of TCDD on overall sperm quality.

\section{Conclusion}

In our study, WGBS of sperm from eight young adults identified 52 DMRs associated with peripubertal serum TCDD concentrations. Our study is the first to support the potential for peripubertal environmental exposures to be associated with subsequent sperm methylation profiles in humans. These findings provide insight into additional likely susceptible windows of development by which environmental exposures can influence epigenetic programming of male germ cells. However, it must be noted that given our small sample size, our results must be interpreted with caution. Future research aims to capitalize on our ongoing prospective study to replicate these initial findings in our larger study population.

\section{Supplementary Material}

Refer to Web version on PubMed Central for supplementary material. 


\section{Acknowledgments}

Funding: This study was supported by Russian Science Foundation (RSF) [grant number 14-45-00065]; parent RCS was supported by US EPA [grant number R82943701] and by NIEHS [grant number R01 ES014370].

We would like to thank the study participants, Chapaevsk government, and the Chapaevsk Medical Association and Chapaevsk Central Hospital staff.

Abbreviations

\begin{tabular}{|c|c|}
\hline AhR & aryl hydrocarbon receptor \\
\hline ARNT & aryl hydrocarbon receptor nuclear translocator \\
\hline DMR & differentially methylated region \\
\hline EDCs & endocrine disrupting chemicals \\
\hline ERa & estrogen receptor alpha \\
\hline ESHRE-SIGA & $\begin{array}{l}\text { European Society of Human Reproduction and } \\
\text { Embryology-Special Interest Group in Andrology }\end{array}$ \\
\hline FDR & false discovery rate \\
\hline G2 & Tanner genitalia stage 2 \\
\hline HPG & hypothalamus-pituitary-gonadal \\
\hline IPA & Ingenuity Pathway Analyses \\
\hline IQR & interquartile range \\
\hline KS & Kolmogorov-Smirnov (test) \\
\hline LOD & limit of detection \\
\hline NAFA & Nordic Association for Andrology \\
\hline P2 & Tanner pubic hair stage 2 \\
\hline PCDDs & polychlorinated dibenzo-p-dioxins \\
\hline $\mathbf{R C S}$ & Russian Children's Study \\
\hline RRBS & reduced representation bisulfite sequencing \\
\hline TCDD & 2,3,7,8-tetrachlorodibenzo-p-dioxin \\
\hline WGBS & whole genome bisulfite sequencing \\
\hline WHO & World Health Organization \\
\hline
\end{tabular}




\section{References}

1. Wu H, Hauser R, Krawetz SA, Pilsner JR. Environmental susceptibility of the sperm epigenome during windows of male germ cell development. Curr Environ Health Rep. 2015a; 2:356-366. [PubMed: 26362467]

2. Manikkam M, Tracey R, Guerrero-Bosagna C, Skinner MK. Dioxin (tcdd) induces epigenetic transgenerational inheritance of adult onset disease and sperm epimutations. PLoS One. 2012; 7:e46249. [PubMed: 23049995]

3. Iqbal K, Tran DA, Li AX, Warden C, Bai AY, Singh P, et al. Deleterious effects of endocrine disruptors are corrected in the mammalian germline by epigenome reprogramming. Genome biology. 2015; 16:59. [PubMed: 25853433]

4. Anway MD, Leathers C, Skinner MK. Endocrine disruptor vinclozolin induced epigenetic transgenerational adult-onset disease. Endocrinology. 2006; 147:5515-5523. [PubMed: 16973726]

5. Guerrero-Bosagna C, Settles M, Lucker B, Skinner MK. Epigenetic transgenerational actions of vinclozolin on promoter regions of the sperm epigenome. PLoS One. 2010; 5

6. Guerrero-Bosagna C, Covert TR, Haque MM, Settles M, Nilsson EE, Anway MD, et al. Epigenetic transgenerational inheritance of vinclozolin induced mouse adult onset disease and associated sperm epigenome biomarkers. Reproductive toxicology (Elmsford, NY). 2012; 34:694-707.

7. Skinner MK, Guerrero-Bosagna C, Haque M, Nilsson E, Bhandari R, McCarrey JR.

Environmentally induced transgenerational epigenetic reprogramming of primordial germ cells and the subsequent germ line. PloS one. 2013; 8:e66318. [PubMed: 23869203]

8. Carone BR, Fauquier L, Habib N, Shea JM, Hart CE, Li R, et al. Paternally induced transgenerational environmental reprogramming of metabolic gene expression in mammals. Cell. 2010; 143:1084-1096. [PubMed: 21183072]

9. Wei Y, Yang CR, Wei YP, Zhao ZA, Hou Y, Schatten H, et al. Paternally induced transgenerational inheritance of susceptibility to diabetes in mammals. Proceedings of the National Academy of Sciences of the United States of America. 2014; 111:1873-1878. [PubMed: 24449870]

10. Mitamura R, Yano K, Suzuki N, Ito Y, Makita Y, Okuno A. Diurnal rhythms of luteinizing hormone, follicle-stimulating hormone, and testosterone secretion before the onset of male puberty. The Journal of clinical endocrinology and metabolism. 1999; 84:29-37. [PubMed: 9920058]

11. Wu FC, Butler GE, Kelnar CJ, Huhtaniemi I, Veldhuis JD. Ontogeny of pulsatile gonadotropin releasing hormone secretion from midchildhood, through puberty, to adulthood in the human male: A study using deconvolution analysis and an ultrasensitive immunofluorometric assay. The Journal of clinical endocrinology and metabolism. 1996; 81:1798-1805. [PubMed: 8626838]

12. Paniagua R, Nistal M. Morphological and histometric study of human spermatogonia from birth to the onset of puberty. J Anat. 1984; 139(Pt 3):535-552. [PubMed: 6490534]

13. Milbrath MO, Wenger Y, Chang CW, Emond C, Garabrant D, Gillespie BW, et al. Apparent halflives of dioxins, furans, and polychlorinated biphenyls as a function of age, body fat, smoking status, and breast-feeding. Environ Health Perspect. 2009; 117:417-425. [PubMed: 19337517]

14. Van den Berg M, Birnbaum LS, Denison M, De Vito M, Farland W, Feeley M, et al. The 2005 world health organization reevaluation of human and mammalian toxic equivalency factors for dioxins and dioxin-like compounds. Toxicological sciences : an official journal of the Society of Toxicology. 2006; 93:223-241. [PubMed: 16829543]

15. WHO. [accessed 20.11.2016] Dioxins and their effects on human health, fact sheet No 225. 2016. Available: http://www.who.int/mediacentre/factsheets/fs225/en/

16. Fernandez-Gonzalez R, Yebra-Pimentel I, Martinez-Carballo E, Simal-Gandara J. A critical review about human exposure to polychlorinated dibenzo-p-dioxins (pcdds), polychlorinated dibenzofurans (pcdfs) and polychlorinated biphenyls (pcbs) through foods. Crit Rev Food Sci Nutr. 2015; 55:1590-1617. [PubMed: 24279584]

17. Minguez-Alarcon L, Sergeyev O, Burns JS, Williams PL, Lee MM, Korrick SA, et al. A longitudinal study of peripubertal serum organochlorine concentrations and semen parameters in young men: The russian children's study. Environ Health Perspect. 2017; 125:460-466. [PubMed: 27713107] 
18. Mocarelli P, Gerthoux PM, Patterson DG Jr, Milani S, Limonta G, Bertona M, et al. Dioxin exposure, from infancy through puberty, produces endocrine disruption and affects human semen quality. Environ Health Perspect. 2008; 116:70-77. [PubMed: 18197302]

19. Pilsner JR, Parker M, Sergeyev O, Suvorov A. Spermatogenesis disruption by dioxins: Epigenetic reprograming and windows of susceptibility. Reproductive toxicology. 2017; 69:221-229. [PubMed: 28286111]

20. Korrick SA, Lee MM, Williams PL, Sergeyev O, Burns JS, Patterson DG, et al. Dioxin exposure and age of pubertal onset among russian boys. Environ Health Perspect. 2011; 119:1339-1344. [PubMed: 21527364]

21. Sergeyev O, Burns JS, Williams PL, Korrick SA, Lee MM, Revich B, et al. The association of peripubertal serum concentrations of organochlorine chemicals and blood lead with growth and pubertal development in a longitudinal cohort of boys: A review of published results from the russian children's study. Reviews on environmental health. 2017; 32:83-92. [PubMed: 28231067]

22. Burns JS, Williams PL, Sergeyev O, Korrick S, Lee MM, Revich B, et al. Predictors of serum dioxins and pcbs among peripubertal russian boys. Environmental health perspectives. 2009; 117:1593-1599. [PubMed: 20019911]

23. Baccarelli A, Pfeiffer R, Consonni D, Pesatori AC, Bonzini M, Patterson DG Jr, et al. Handling of dioxin measurement data in the presence of non-detectable values: Overview of available methods and their application in the seveso chloracne study. Chemosphere. 2005; 60:898-906. [PubMed: 15992596]

24. Björndahl L, Mortimer D, Barratt CLR, Castilla JA, Menkveld R, Kvist U., et al. A practical guide to basic laboratory andrology. Cambridge, UK: Cambridge University Press; 2010.

25. Kvist U, Bjorndahl L. Eshre monographs. Oxford: Oxford University Press; 2002. Manual on basic semen analysis.

26. World Health Organization. Who laboratory manual for the examination of human semen and sperm-cervical mucus interaction. 4. Cambridge, United Kingdom: Cambridge University Press; 1999.

27. Wu H, de Gannes M, Luchetti G, Pilsner JR. Rapid method for the isolation of mammalian sperm DNA. Biotechniques. 2015b; 58:293-300. [PubMed: 26054765]

28. Krueger F, Andrews SR. Bismark: A flexible aligner and methylation caller for bisulfite-seq applications. Bioinformatics (Oxford, England). 2011; 27:1571-1572.

29. Langmead B, Salzberg SL. Fast gapped-read alignment with bowtie 2. Nature methods. 2012; 9:357-359. [PubMed: 22388286]

30. ENCODE. [accessed September 2, 2016] Encode and modencode guidelines for experiments generating chip, dnase, faire, and DNA methylation genome wide location data. Version 2.0. 2011. Available: http://genome.ucsc.edu/ENCODE/protocols/dataStandards/ ChIP_DNase_FAIRE_DNAme_v2_2010.pdf

31. Song Q, Decato B, Hong EE, Zhou M, Fang F, Qu J, et al. A reference methylome database and analysis pipeline to facilitate integrative and comparative epigenomics. PLoS One. 2013; 8:e81148. [PubMed: 24324667]

32. Neph S, Kuehn MS, Reynolds AP, Haugen E, Thurman RE, Johnson AK, et al. Bedops: Highperformance genomic feature operations. Bioinformatics (Oxford, England). 2012; 28:1919-1920.

33. Karolchik D, Hinrichs AS, Furey TS, Roskin KM, Sugnet CW, Haussler D, et al. The ucsc table browser data retrieval tool. Nucleic acids research. 2004; 32:D493-496. [PubMed: 14681465]

34. Andersson R, Gebhard C, Miguel-Escalada I, Hoof I, Bornholdt J, Boyd M, et al. An atlas of active enhancers across human cell types and tissues. Nature. 2014; 507:455-461. [PubMed: 24670763]

35. Lizio M, Harshbarger J, Shimoji H, Severin J, Kasukawa T, Sahin S, et al. Gateways to the fantom5 promoter level mammalian expression atlas. Genome biology. 2015; 16:22. [PubMed: 25723102]

36. Kent WJ, Sugnet CW, Furey TS, Roskin KM, Pringle TH, Zahler AM, et al. The human genome browser at ucsc. Genome research. 2002; 12:996-1006. [PubMed: 12045153]

37. Rosenbloom KR, Armstrong J, Barber GP, Casper J, Clawson H, Diekhans M, et al. The ucsc genome browser database: 2015 update. Nucleic acids research. 2015; 43:D670-681. [PubMed: 25428374] 
38. Donkin I, Versteyhe S, Ingerslev LR, Qian K, Mechta M, Nordkap L, et al. Obesity and bariatric surgery drive epigenetic variation of spermatozoa in humans. Cell metabolism. 2016; 23:369-378. [PubMed: 26669700]

39. Jones EOE, Peterson P. , et al. [accessed April 20 2017] Scipy: Open source scientific tools for python. 2001. Available: http://www.scipy.org/

40. Arpanahi A, Brinkworth M, Iles D, Krawetz SA, Paradowska A, Platts AE, et al. Endonucleasesensitive regions of human spermatozoal chromatin are highly enriched in promoter and ctcf binding sequences. Genome research. 2009; 19:1338-1349. [PubMed: 19584098]

41. Hammoud SS, Nix DA, Zhang H, Purwar J, Carrell DT, Cairns BR. Distinctive chromatin in human sperm packages genes for embryo development. Nature. 2009; 460:473-478. [PubMed: 19525931]

42. Lambrot R, Xu C, Saint-Phar S, Chountalos G, Cohen T, Paquet M, et al. Low paternal dietary folate alters the mouse sperm epigenome and is associated with negative pregnancy outcomes. Nature communications. 2013; 4:2889.

43. Radford EJ, Ito M, Shi H, Corish JA, Yamazawa K, Isganaitis E, et al. In utero effects. In utero undernourishment perturbs the adult sperm methylome and intergenerational metabolism. Science. 2014; 345:1255903. [PubMed: 25011554]

44. Sharma U, Conine CC, Shea JM, Boskovic A, Derr AG, Bing XY, et al. Biogenesis and function of trna fragments during sperm maturation and fertilization in mammals. Science. 2016; 351:391396. [PubMed: 26721685]

45. Schlatt S, Ehmcke J. Regulation of spermatogenesis: An evolutionary biologist's perspective. Semin Cell Dev Biol. 2014; 29:2-16. [PubMed: 24685618]

46. Vergouwen RP, Huiskamp R, Bas RJ, Roepers-Gajadien HL, Davids JA, de Rooij DG. Postnatal development of testicular cell populations in mice. J Reprod Fertil. 1993; 99:479-485. [PubMed: 8107030]

47. Lee MM, Donahoe PK, Hasegawa T, Silverman B, Crist GB, Best S, et al. Mullerian inhibiting substance in humans: Normal levels from infancy to adulthood. The Journal of clinical endocrinology and metabolism. 1996; 81:571-576. [PubMed: 8636269]

48. Muller J, Skakkebaek NE. Quantification of germ cells and seminiferous tubules by stereological examination of testicles from 50 boys who suffered from sudden death. International journal of andrology. 1983; 6:143-156. [PubMed: 6862671]

49. Mocarelli P, Gerthoux PM, Needham LL, Patterson DG Jr, Limonta G, Falbo R, et al. Perinatal exposure to low doses of dioxin can permanently impair human semen quality. Environ Health Perspect. 2011; 119:713-718. [PubMed: 21262597]

50. Burns JS, Lee MM, Williams PL, Korrick SA, Sergeyev O, Lam T, et al. Associations of peripubertal serum dioxin and polychlorinated biphenyl concentrations with pubertal timing among russian boys. Environ Health Perspect. 2016; 124:1801-1807. [PubMed: 27187981]

51. Humblet O, Korrick SA, Williams PL, Sergeyev O, Emond C, Birnbaum LS, et al. Genetic modification of the association between peripubertal dioxin exposure and pubertal onset in a cohort of russian boys. Environ Health Perspect. 2013; 121:111-117. [PubMed: 23060366]

52. Safe S, Wormke M. Inhibitory aryl hydrocarbon receptor-estrogen receptor alpha cross-talk and mechanisms of action. Chem Res Toxicol. 2003; 16:807-816. [PubMed: 12870882]

53. Swedenborg E, Pongratz I. Ahr and arnt modulate er signaling. Toxicology. 2010; 268:132-138. [PubMed: 19778576]

54. Beischlag TV, Perdew GH. Er alpha-ahr-arnt protein-protein interactions mediate estradioldependent transrepression of dioxin-inducible gene transcription. The Journal of biological chemistry. 2005; 280:21607-21611. [PubMed: 15837795]

55. Ohtake F, Takeyama K, Matsumoto T, Kitagawa H, Yamamoto Y, Nohara K, et al. Modulation of oestrogen receptor signalling by association with the activated dioxin receptor. Nature. 2003; 423:545-550. [PubMed: 12774124]

56. Carreau S, Hess RA. Oestrogens and spermatogenesis. Philosophical transactions of the Royal Society of London Series B, Biological sciences. 2010; 365:1517-1535. [PubMed: 20403867]

57. Hess RA, Bunick D, Lee KH, Bahr J, Taylor JA, Korach KS, et al. A role for oestrogens in the male reproductive system. Nature. 1997; 390:509-512. [PubMed: 9393999] 
58. Joseph A, Hess RA, Schaeffer DJ, Ko C, Hudgin-Spivey S, Chambon P, et al. Absence of estrogen receptor alpha leads to physiological alterations in the mouse epididymis and consequent defects in sperm function. Biology of reproduction. 2010a; 82:948-957. [PubMed: 20130267]

59. Joseph A, Shur BD, Ko C, Chambon P, Hess RA. Epididymal hypo-osmolality induces abnormal sperm morphology and function in the estrogen receptor alpha knockout mouse. Biology of reproduction. 2010b; 82:958-967. [PubMed: 20130266]

60. Nanjappa MK, Hess RA, Medrano TI, Locker SH, Levin ER, Cooke PS. Membrane-localized estrogen receptor 1 is required for normal male reproductive development and function in mice. Endocrinology. 2016; 157:2909-2919. [PubMed: 27145009]

61. Chimento A, Sirianni R, Zolea F, Bois C, Delalande C, Ando S, et al. Gper and esrs are expressed in rat round spermatids and mediate oestrogen-dependent rapid pathways modulating expression of cyclin b1 and bax. International journal of andrology. 2011; 34:420-429. [PubMed: 20969598]

62. Chimento A, Sirianni R, Casaburi I, Ruggiero C, Maggiolini M, Ando S, et al. 17beta-estradiol activates gper- and esr1-dependent pathways inducing apoptosis in gc-2 cells, a mouse spermatocyte-derived cell line. Molecular and cellular endocrinology. 2012; 355:49-59. [PubMed: 22306083]

63. Boussouar F, Goudarzi A, Buchou T, Shiota H, Barral S, Debernardi A, et al. A specific cbp/p300dependent gene expression programme drives the metabolic remodelling in late stages of spermatogenesis. Andrology. 2014; 2:351-359. [PubMed: 24522976]

64. Hammoud SS, Nix DA, Hammoud AO, Gibson M, Cairns BR, Carrell DT. Genome-wide analysis identifies changes in histone retention and epigenetic modifications at developmental and imprinted gene loci in the sperm of infertile men. Human Reproduction. 2011; 26:2558-2569. [PubMed: 21685136]

65. Wykes SM, Krawetz SA. The structural organization of sperm chromatin. The Journal of biological chemistry. 2003; 278:29471-29477. [PubMed: 12775710]

66. Carone BR, Hung JH, Hainer SJ, Chou MT, Carone DM, Weng Z, et al. High-resolution mapping of chromatin packaging in mouse embryonic stem cells and sperm. Developmental cell. 2014; 30:11-22. [PubMed: 24998598]

67. Samans B, Yang Y, Krebs S, Sarode GV, Blum H, Reichenbach M, et al. Uniformity of nucleosome preservation pattern in mammalian sperm and its connection to repetitive DNA elements. Developmental cell. 2014; 30:23-35. [PubMed: 24998597]

68. Shea JM, Serra RW, Carone BR, Shulha HP, Kucukural A, Ziller MJ, et al. Genetic and epigenetic variation, but not diet, shape the sperm methylome. Developmental cell. 2015; 35:750-758. [PubMed: 26702833]

69. Kobayashi H, Sato A, Otsu E, Hiura H, Tomatsu C, Utsunomiya T, et al. Aberrant DNA methylation of imprinted loci in sperm from oligospermic patients. Human molecular genetics. 2007; 16:2542-2551. [PubMed: 17636251]

70. Marques CJ, Costa P, Vaz B, Carvalho F, Fernandes S, Barros A, et al. Abnormal methylation of imprinted genes in human sperm is associated with oligozoospermia. Molecular human reproduction. 2008; 14:67-74. [PubMed: 18178607]

71. Pacheco SE, Houseman EA, Christensen BC, Marsit CJ, Kelsey KT, Sigman M, et al. Integrative DNA methylation and gene expression analyses identify DNA packaging and epigenetic regulatory genes associated with low motility sperm. PLoS One. 2011; 6:e20280. [PubMed: 21674046] 


\section{Highlights}

- Whole genome bisulfite sequencing of sperm in young adults was conducted to examine associations with serum dioxin concentrations at $8-9$ years

- $\quad 52$ differentially methylated regions (DMRs) were identified that distinguished lowest and highest peripubertal serum TCDD concentrations

- $\quad$ Peripubertal period is susceptible windows of development by which environmental exposures can influence epigenetic programming of male germ cells 
A

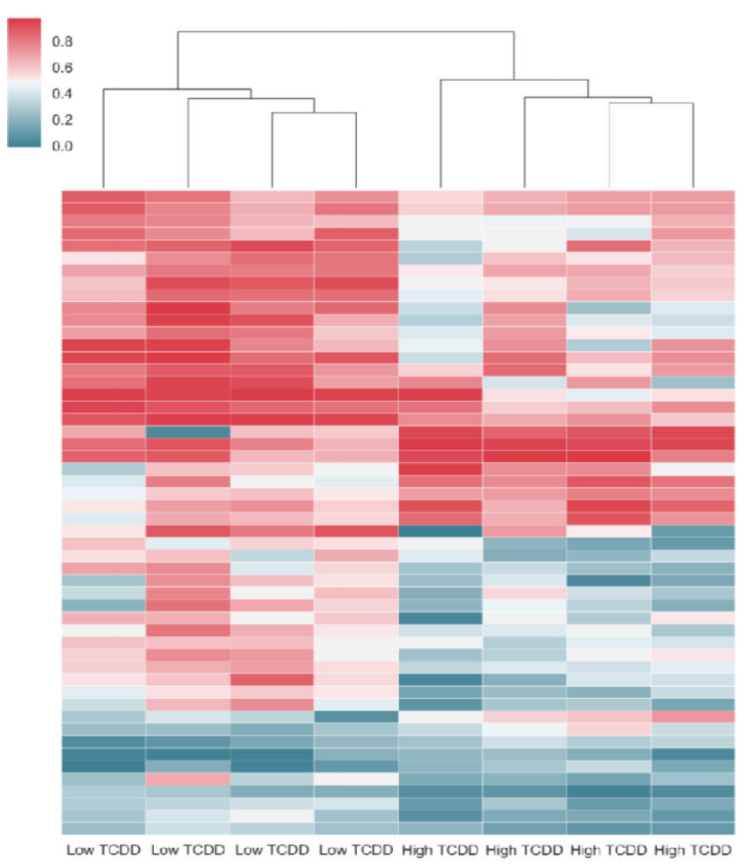

C

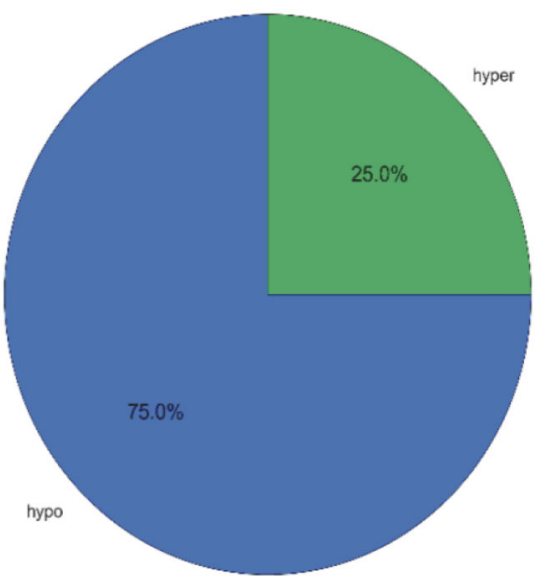

B

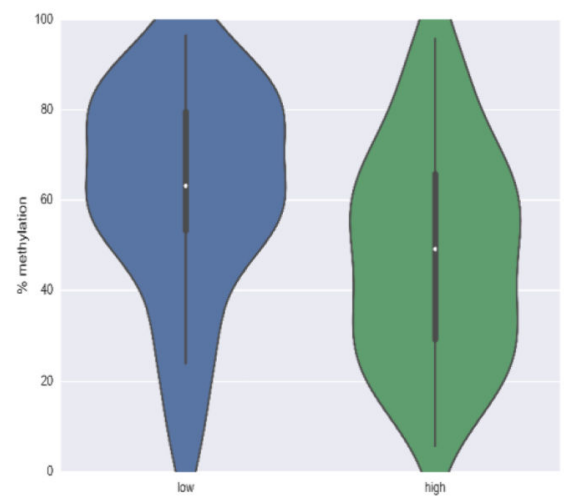

D

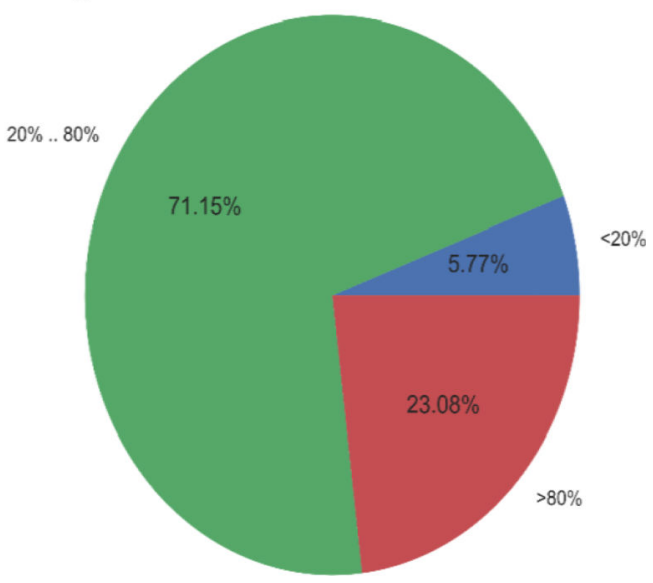

Figure 1.

Differential methylated regions (DMRs) in sperm associated with highest peripubertal serum 2,3,7,8-tetrachlorodibenzo-p-dioxin (TCDD) concentrations.

A. Unsupervised hierarchical clustering and heatmap of the 52 significant DMRs of individuals with lowest and highest peripubertal TCDD groups. High percent methylation is shown in red while low percent methylation is in blue.

B. Violin plot of the median and interquartile range (black box plot) and distribution of average methylation of the 52 DMRs between TCDD groups. 
C. Pie chart of the percent of DMRs displaying hypermethylation and hypomethylation in comparison to lowest TCDD group.

D. Pie chart displaying percent methylation of DMRs with low $(<20 \%)$, intermediate $(20-$ $80 \%)$ or high (>80\%) average methylation. 

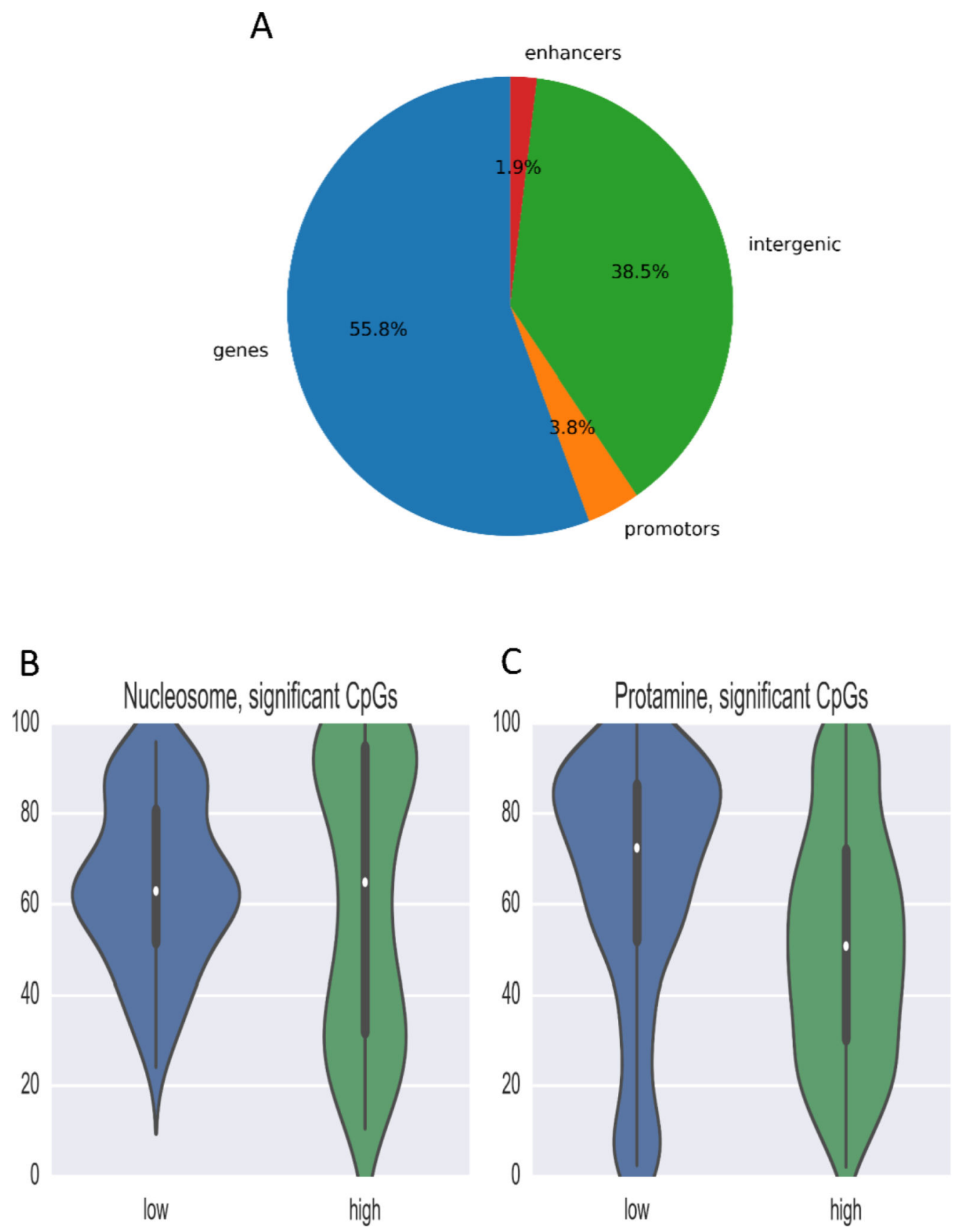

Figure 2.

Annotation of sperm differential methylated regions (DMRs) or individual $\mathrm{CpG}$ sites in sperm associated with highest peripubertal serum 2,3,7,8-tetrachlorodibenzo-p-dioxin (TCDD) concentrations.

A. Pie chart of the relative proportion of significant DMRs based on genomic locations.

B. Violin plots of nucleosome-associated significant CpGs by lowest and highest peripubertal serum TCDD groups.

C. Violin plots of protamine-associated significant CpGs by lowest and highest peripubertal serum TCDD groups. 

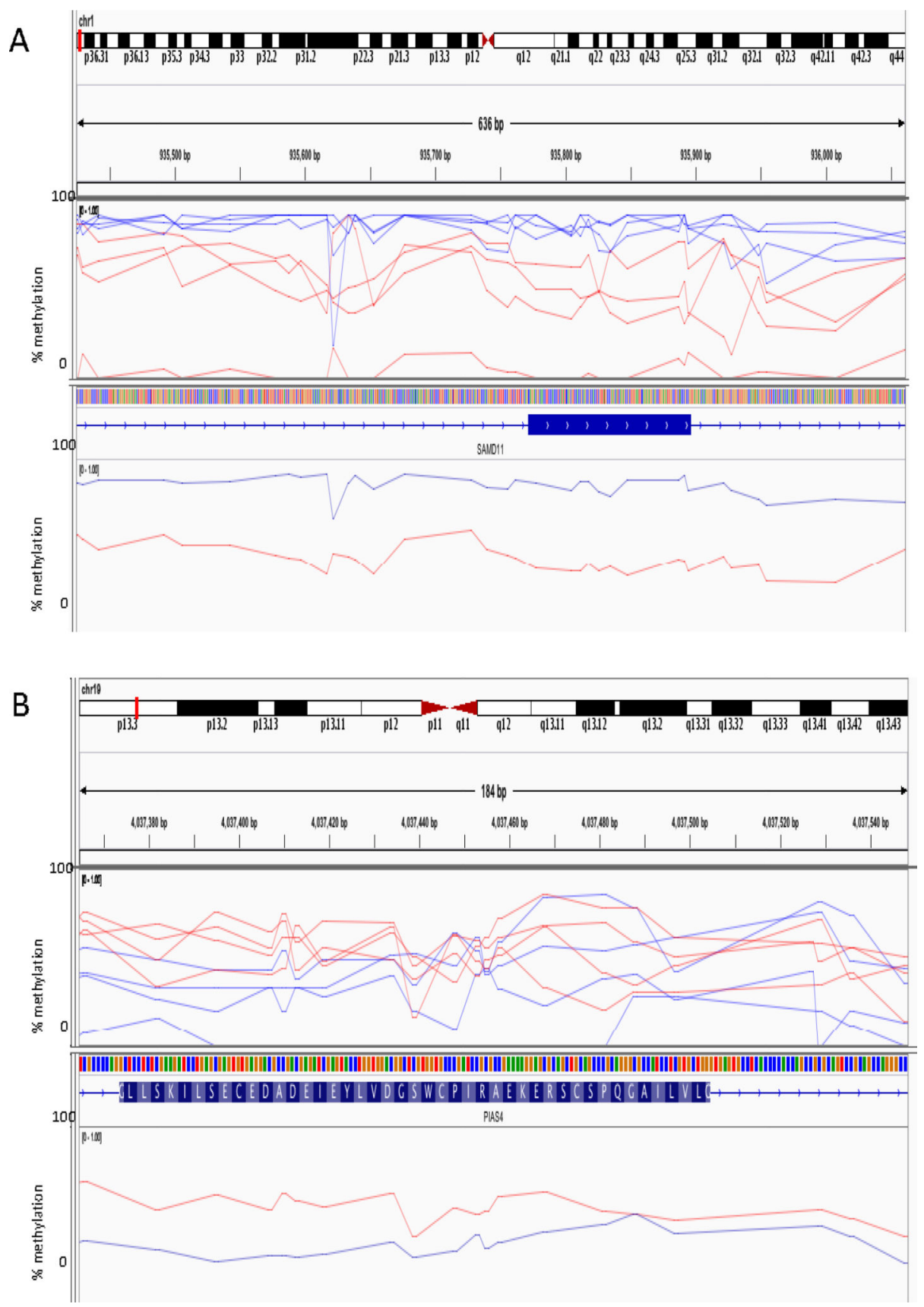

Figure 3.

Illustration of sperm differential methylated regions (DMRs) associated with highest peripubertal 2,3,7,8-tetrachlorodibenzo-p-dioxin (TCDD) concentrations.

A. Integrative Graphical View (IGV) of percent methylation (y-axis) for the nucleosomeassociated SAMD11 (CpGs $=20$ ) of individual participants (top panel) and average (bottom panel) by lowest (red lines) and highest (blue lines) peripubertal serum TCDD groups. B. IGV of percent methylation (y-axis) for the protamine-associated PIAS4 (CpGs $=8$ ) of individual participants (top panel) and average (bottom panel) by lowest (red lines) and highest (blue lines) peripubertal serum TCDD groups. 


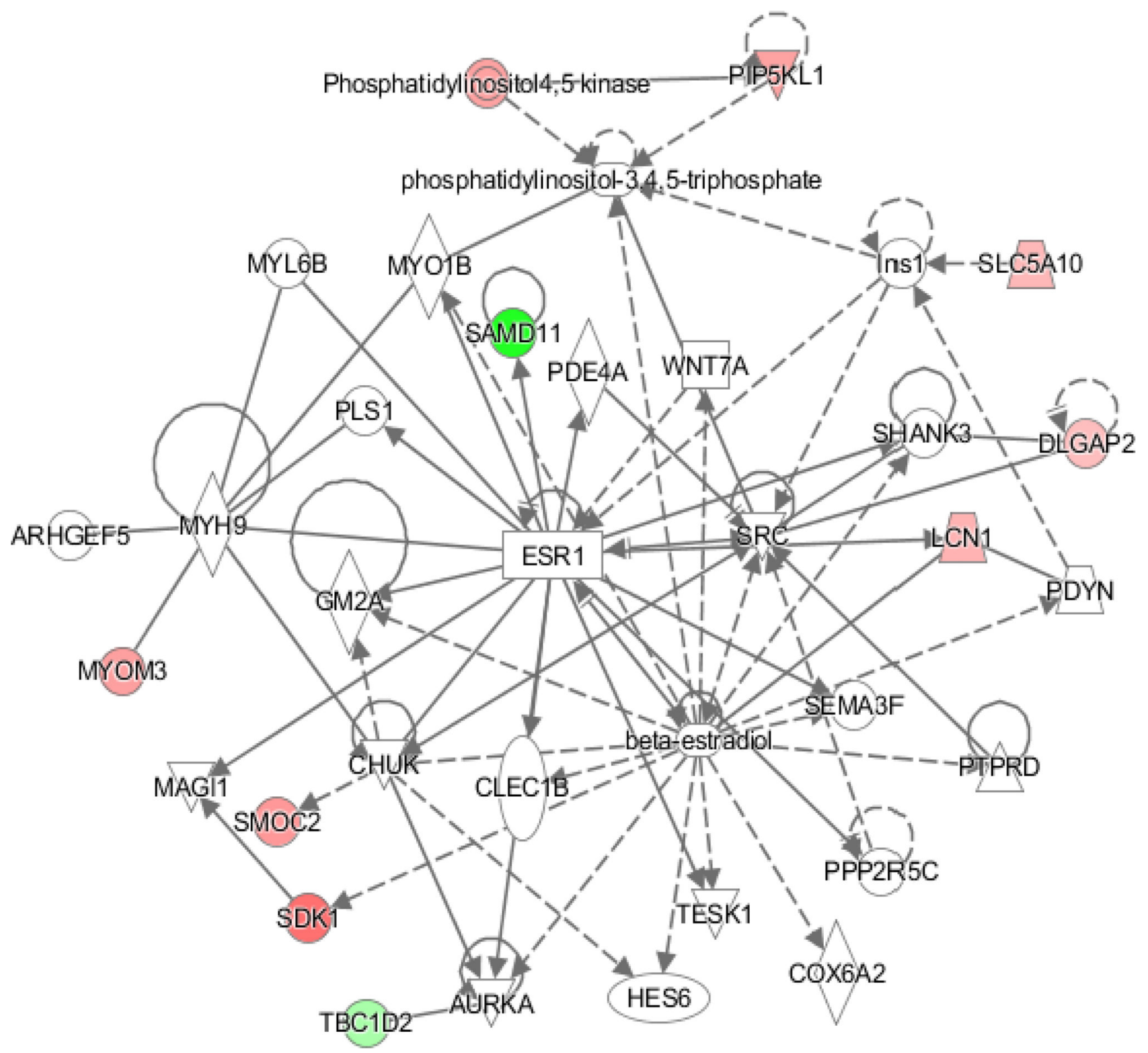

Figure 4.

One of top scored networks showing the estrogen receptor alpha (ERa/ESR1) as the central regulator under the function of "Cellular Assembly and Organization, Cellular Function and Maintenance, Carbohydrate Metabolism".

Genes are represented by nodes with their shape representing the type of molecule/ functional class. Nodes in red are hypomethylated while green are hypermethylated in highest compared to lowest serum 2,3,7,8-tetrachlorodibenzo-p-dioxin (TCDD) group. 


\section{Table 1}

Descriptive characteristics [mean (range)] of 8 young adults with highest and lowest 2,3,7,8tetrachlorodibenzo-p-dioxin (TCDD) peripubertal exposure selected for whole genome bisulfite sequencing in the Russian Children's Study.

\begin{tabular}{|c|c|c|}
\hline \multirow[b]{2}{*}{ Characteristic } & \multicolumn{2}{|c|}{ TCDD peripubertal concentration } \\
\hline & $\begin{array}{c}\text { Lowest TCDD } \\
(N=4)\end{array}$ & $\begin{array}{c}\text { Highest TCDD } \\
\quad(N=4)\end{array}$ \\
\hline \multicolumn{3}{|l|}{ Dioxins serum levels at study entry } \\
\hline TCDD, pg/g lipid & $0.39^{a}(0.35-0.49)$ & $10.4(8-12.1)$ \\
\hline Total TEQ, pg TEQ/g lipid & $11.1^{a}(6.6-16.0)$ & $56.4(26.2-76.1)$ \\
\hline \multicolumn{3}{|c|}{ Age and anthropometric measures at semen collection } \\
\hline Age, years & $18.8(18.1-19.3)$ & $18.7(18.1-19.1)$ \\
\hline Weight, $\mathrm{kg}$ & $74.5(67-79.2)$ & $67.4(58-76.1)$ \\
\hline Height, cm & $183.5(181.4-186)$ & $179.1(174-186.7)$ \\
\hline BMI, $\mathrm{kg} / \mathrm{m}^{2}$ & $22.1(19.4-23.6)$ & $21.0(18.7-23.6)$ \\
\hline \multicolumn{3}{|c|}{ Smoking and alcohol consumption last year before semen collection, n (\%) } \\
\hline Smoking status $b$ & $0(0)$ & $0(0)$ \\
\hline Alcohol consumption ${ }^{c}$ & $1(25)$ & $3(100)^{d}$ \\
\hline \multicolumn{3}{|l|}{ Nutrition at study entry, $8-9$ years old } \\
\hline Total daily dietary intake, kcal & $2828(2059-3539)$ & $3353(2716-4011)$ \\
\hline Percent daily calories from protein, $\%$ & $12.8(10.4-15.0)$ & $11.9(10.4-13.7)$ \\
\hline \multicolumn{3}{|l|}{ Sexual maturity and semen parameters } \\
\hline Mean testicular volume, $\mathrm{ml}$ & $24.4(20-28.8)$ & $23.1(17.5-28.8)$ \\
\hline Abstinence duration, hours & $166.3(31-480)$ & $51.5(37-63)$ \\
\hline Semen volume, $\mathrm{ml}$ & $3.9(0.8-9.2)$ & $5.0(1.5-7.9)$ \\
\hline Motility, a+b+c, \% & $59.3(48-66)$ & $65.3(64-68)$ \\
\hline Sperm concentration, $\mathrm{ml} / \mathrm{mill}$ & $89.8(53-115.6)$ & $49.3(13.9-82.3)$ \\
\hline Total motile sperms, mill & $170.1(34.2-251.8)$ & $105.0(68.5-144.3)$ \\
\hline Total sperm count, mill & $293(60-487.6)$ & $161.4(107-222.1)$ \\
\hline \multicolumn{3}{|c|}{${ }^{a}$ Two-sided Wilcoxon rank-sum test with P-value $₫$. 05} \\
\hline \multicolumn{3}{|c|}{$b$ Question was "In the past year, have you smoked a cigarette, even a few puffs?" } \\
\hline
\end{tabular}




\section{Table 2}

Functional networks generated by Ingenuity Pathway Analyses (IPA) of significant sperm differentially methylated regions associated with peripubertal 2,3,7,8-tetrachlorodibenzo-p-dioxin (TCDD) exposure.

\begin{tabular}{|c|c|l|}
\hline $\begin{array}{c}\text { IPA Network } \\
\text { Score }\end{array}$ & $\begin{array}{c}\text { Focus } \\
\text { Molecules }\end{array}$ & Top Diseases and Functions \\
\hline 19 & 9 & Cell Morphology, Hematopoiesis, Humoral Immune Response \\
\hline 19 & 9 & Cellular Assembly and Organization, Cellular Function and Maintenance, Carbohydrate Metabolism \\
\hline 19 & 9 & DNA Replication, Recombination, and Repair, Energy Production, Nucleic Acid Metabolism \\
\hline 7 & 4 & Embryonic Development, Organismal Development, Tissue Development \\
\hline 3 & 1 & Infectious Diseases, Cell Death and Survival, Protein Synthesis \\
\hline 2 & 1 & Post-Translational Modification, Cellular Movement, Respiratory System Development and Function \\
\hline
\end{tabular}

